\title{
Forecasting Price Direction, Hedging and Spread Options in Oil Volatility
}

\author{
Eboka Andrew Okonji, Yerokun Oluwatoyin Mary \\ Department of Computer Education, Federal College of Education Technical, Asaba, Nigeria
}

\section{Email address:}

an_drey2k@yahoo.com (E. A. Okonji), agapenexus@hotmail.com (Y. O. Mary)

\section{To cite this article:}

Eboka Andrew Okonji, Yerokun Oluwatoyin Mary. Forecasting Price Direction, Hedging and Spread Options in Oil Volatility. International Journal of Economic Behavior and Organization. Vol. 5, No. 6, 2017, pp. 114-123. doi: 10.11648/j.ijebo.20170506.11

Received: April 5, 2017; Accepted: October 8, 2017; Published: November 8, 2017

\begin{abstract}
The energy market aims to manage risks associated with prices and volatility of the asset. It is a capital intensive market, rippled with a range of chaotic, complex and dynamic interaction among its supply and demand derivatives. Models help users forecast such interactions, to provide investors with empirical evidence of the price direction. Evolutionary modeling is an art, whose science seeks to analyze input data and yield an optimal, complete solution for which conventional methods yield a corresponding, non-cost effective solution. Its solutions are tractable, robust and low-cost with a tolerance of ambiguity, uncertainty and noise as applied to its input. Our study aims to predict the OPEC Oil market with data collected over the period.
\end{abstract}

Keywords: Energy, OPEC, Stochastic, Evolutionary Model, Price Direction, Volatility

\section{Introduction}

The asset market has now become a focal point for diversification in the finance world with the energy market playing a dominant and crucial role. With an increased supply/demand, and heavy dependence on oil has also brought about a number of complexities ranging from production, storage, transportation and stringent regulation issue, all of which continue to plague its effective management (Laurenti and Fernandes, 2012). Market participants now invest, knowing that such asset yields interesting diversification benefits, significant reward cum positive investment returns to their financial portfolio (Ojugo, 2016) because oil currently accounts for over $10 \%$ of the actively traded assets and is the world's largest consumed asset (Verleger, 1993). Investors and researchers must continue to seek effective means of futures trading via its supply/demand parameters (Ojugo and Ofualagba, 2016) so that empirical results based on their analytic findings can help further dispose participants to the Oil (energy) market.

The recent plummet in price, seem like an indication to the end of the Oil-era. But, studies show (as in figure 2) that the global demand of oil continues to rise despite that demands from the Organization for Economic Co-operation and Development (OECD) nations has decreased. But, OPEC data shows that Oil's overall demand is increasing with demands from non-OECD nations like China (EIA Report, 2009); And, that a significant amount of oil is supplied from 'unstable' Middle East connotes that more price fluctuations is to be welcomed as normal. Thus, prediction of oil price direction is useful for investors and market participants (Ojugo, 2016).

It is common knowledge that taking a position in spot market, is not the best way to react to new data as such decision is besieged by high cost of transaction, storage costs, inconveniences, high premium and delivery costs among others, etc - especially if the investor is not interested in such asset; But, rather hedging for another asset and/or speculating by simply investing in hope of arbitrage opportunity. Thus, the futures market is more attractive because an investor can react to new data for the right reason (Silvapulle and Moosa, 1999).

A cursory look at many studies showcases varied inconsistencies and discrepancies in their findings relating to spot- and futures-price. And whilst many of these studies agree on the importance of futures prices; only a few agree how important it is, as majority of the studies are based on analytic models.

Bopp and Sitzer (1987) reveals that while futures price can predict cash price via improving the forecasts in econometrical models, only future contract of 1- to 2-months maturity are statistically significant as to contain new data. Also, Chan (1992) and Abosedra and Baghestani (2004) concurred that the cash market is a firm source of new data 
and that futures price 1- to 12-months ahead yields significant forecast and useful for policy making.

Moshiri and Foroutan (2005) examined the chaotic and nonlinear feat in futures prices, comparing ARMA/GARCH linear models against the nonlinear ANN model. Result showed ANN is statistically more significant and outperformed both ARMA and GARCH as futures price is stochastic and nonlinear. But, Kulkarni and Haidar (2009) observed 2-errors in the study: (a) use of raw data input in ANN, and (b) training data is quite old for the period used.

Coppola (2007) proved that futures contracts reflects data about spot price's future direction; while Wang et al (2005) in their hybrid model, merged web-mining for rule extraction, ANN and ARIMA model and claims the nonlinear integration of these 3-models outperforms any single model. But, Kulkarni and Haidar (2009) notes: (1) the controversial, unreliable nature of its rule-base system depends on a knowledgebase designed by expert czar as even many experts' opinion(s) vary on the same task, and (2) neither the knowledgebase nor its corresponding rules were made available for further verification.

Kulkarni and Haidar (2009) used ANN to predict price and volatility. They claimed their model performed well; But, we note a systemic errors in their design using feedforward network. For feedforward nets, all data are treated as new. Such new data become historic data after some iteration, and should not be used (as in their claim) since it cannot help the network identify feats of interest. In broadening our data length coverage, we treat all data (previous and current) as input for in-sample forecast, even if the data exhibit temporal dependence. A major error in their design is that as network grows larger via adding more data, feedforward network are practically difficult to implement (Ojugo et al, 2013a, b).

Our study presents GANN model to predict price and volatilities of oil for short term structure using Gabillon model as preprocessor. Section 2 presents methodology, Section 3 presents our model design, Section 4 details result and discussion, and Section 5 concludes the study.

\section{Methodology}

\subsection{Motivation}

Why must we keep re-investigating prediction of oil futures price; while a plethora of studies and models to accomplish such, are available to us? We draw our statement of problem as thus:

1. Plummet in 'expected-rise' of futures price implies that previous studies did a poor forecast. We need to know why and how it happened.

2. Future price is a continuous 'inconclusive' task and its forecast grants us insights into expected values as the continued enhancement of futures price, update of sample-period and broadening of data coverage to enhance forecasts - will all aim to bring us closer to a definitive answer.

3. Price forecast is crucial; But, costly. For policymakers in countries where price significantly affects governance policies and many other activities, obtaining the best possible forecast is of paramount importance and priority.

4. The chaotic, volatile nature of oil market along with its range of complications makes imperative, early and accurate prediction to help dispose participants properly. Careful observance of spot prices alone, is insufficient as unknown input and inconclusive results may elude them. Thus, without careful analysis, this will lead to increased false-positive and true-negative rates classification activities (resolved in Section 3).

5. Its data consist of ambiguities, impartial truth and noise - which must be resolved via a robust search so as to effectively classify observations and expected values, as in Section 3.

6. Prediction in non-linear models is complex and difficult due to the chaotic and dynamic nature of task, and of unsupervised learning adopted in the models. And use of hill-climbing method further imposes speed constraint on NN so that its solutions are often trapped at local maxima. This is resolved with hybridization as the search for optima via hybrid is better than any single method. But, our model must be able to resolve statistical dependencies imposed on it by hybrid method and dataset (resolved in Section 3).

7. Search for solution can often lead to overtraining, over-fitting and over-parameterization (inadequate and improper selection of parameter) of a model if a lesser data coverage is used. A larger dataset helps the model in its generalization as it seeks underlying probability in data feat(s) of interest as resolved in Section 3.

The proposed genetic algorithm trained neural network aims to predict futures price and volatility as it propagates data (observed/current) to seek feats of interest. It chooses its output from a set of possible solution space data, to yield an output that is void of noise, ambiguities and guaranteed of high quality (Ojugo et al, 2013). The model can be further tuned to be robust so as to perform quantitative processing and ensure qualitative knowledge as its new language (Heppner and Grenander, 1990).

\subsection{Gabillon Model as Preprocessor}

Gabillon (1991) extended Gibson and Schwartz (1990) model for futures price forecast. The model assumes futures price depends on: (a) spot price of Oil and (b) cost to carry the physical oil. Investor's attitude towards the spot price risk(s) and expected increase in spot price are irrelevant to the pricing of a futures contract on Oil. Spot price is given by Eq. 2 where $\mu(\mathrm{S})$ is mean (expected drift rate per unit in time) and $\sigma(\mathrm{S})$ is standard deviation (volatility of the process), and $\mathrm{dz}$ is Wiener process given by:

$$
d S=\mu(S) d t+\sigma(S) d z
$$

The futures price for short-term, independent of the stochastic process of the spot price with $r$ as riskless interest 
rate, $\mathrm{C}_{\mathrm{c}}$ as marginal carry cost, $\mathrm{C}_{\mathrm{y}}$ as marginal convenience yield and $\mathrm{C}_{\mathrm{p}}$ is marginal influence yield, as given by:

$$
F(S, z)=S e^{\left(r+C_{c}-C_{y}+C_{p}\right)^{z}}
$$

We include $C_{p}$ shock for these reasons: (a) energy is about dominance. Nations seek to less dependent on others, for the more a nation depends on another - the more influence such nation she depends on, exerts her politics and policies over her, and (b) this creates new frontier for international politics with franchises made, nation policy interest aligned, treaties brokered; And thus, leads to off-channel sales via diversion tactics from non-OPEC nations, non-observance in limit placed by regulatory bodies like OPEC etc.

\subsection{Artificial Neural Network (ANN)}

ANN consists of interconnected neurons that learn via example (Abraham, 2005). As it processes data, its neurons share data and adjust their weights and bias via firing, to strengthen connection between its synapses (Caudill, 1987; Fausett, 1994). As learning occurs, its weights and biases are adjusted and data is converted via its activation function, which modulates its associated input to learn the nonlinear feats exhibited in the model data and yields an output (Mandic and Chambers, 2001) as in Eq. 3:

$$
\emptyset=f(n e t)=f \sum_{i=1}^{m} X i * W i j
$$

It thus attempts to translate into mathematical model, biological processing principles and generate in its fastest time, the predictive outcome of a task. It derives possible outcomes from experience and recognizes behavioural feats of interest from historic data - to suggest an optimal solution of high quality and void of over-fitting, irrespective of modification made to it via other approximations with multiple agents (Dawson and Wilby 2001a). It connections are set with either apriori, or post-priori knowledge that trains the network to learn patterns that changes its weight and bias based on a rule (Beven and Binley, 1992; Bishop, 1995). Learning is grouped into: supervised, unsupervised and reinforcement, which is encoded via its input, hidden and output layers to make for two configurations: feedforward net where data flows directly from input to output and extends over multiple layers), or recurrent net has feedback with dynamic feats to evolve the net as it undergoes relaxation to a stable state where its output changes no more. In some task, if output change is significant - then dynamic behaviour yields its output (Ojugo et al, 2013b).

The nature of oil market is such that its prediction also requires previous knowledge. Thus, we adopt recurrent (Jordan) net to incorporate previous dataset feats of interest as input variables into model. Thus, it allows previous dataset and previous output to be feedback as input into model's hidden units (Rajurkar et al, 2004; Karunanthis et al, 1994) to yield an output. Its correlated weights are interconnected with $\mathrm{W}_{i . j}$ as weight between input and hidden layers, $\mathrm{W}_{o} \mathrm{j}$ is bias and $\mathrm{x}_{i}$ is market input data sent to yield an output via tangent/sigmoid transfer function as in Eq. 4 and Eq. 5 (Minns, 1998; Chakraborty, 2010).

$$
\begin{aligned}
Z_{i j} & =w_{o} j+\sum_{i=1}^{m} x_{i} * w_{i} j \\
F\left(Z_{i j}\right) & =\frac{2}{1+e^{-2 * Z i j}}-1
\end{aligned}
$$

Our Jordan net is constructed by adding a context layer to (modify) a multilayered feedforward. This will help it retain data between iterations. On start of the algorithm, the context layer is initialized to zero so that output from first iteration is fed back as input into hidden layer (Perez and Marwala, 2011) - so that for the next time step, previous contents of the hidden layer are then passed unto context layer, which helps to yield a new input that is also resent back again as into hidden layer in another time-step (Regianni and Rientjes, 2005). Weights are recomputed in same manner for new connections fro/to its context layer from hidden layer. And, training aims at best fit data weights computed via Tansig function, which assumes an approximation influence of data points at its center. Thus, the function decreases with distance from its center. Its Euclidean length $\left(\mathrm{r}_{\mathrm{j}}\right)$ is distance between vector $\mathrm{y}=\left(\mathrm{y}_{1}, \ldots, \mathrm{y}_{\mathrm{m}}\right)$ and center $\left(\mathrm{w}_{l j}, \ldots, \mathrm{w}_{m j}\right)$ as:

$$
r_{j}=|| y-Y^{j}||=\left\{\sum_{i=1}^{m}\left(y_{i}-w_{i} j\right)^{2}\right\}^{1 / 2}
$$

The suitable transfer function is applied to $r_{j}$ :

$$
\varnothing\left(r_{j}\right)=\varnothing \| y-Y^{j}||
$$

Finally, output k receives weighted combination as:

$$
y^{k}=w_{o}+\sum_{j=1}^{n}\left(c_{j}^{k} * \emptyset\left(r_{j}\right)\right)=w_{o}+\sum_{j=1}^{n}\left(c_{j}^{k} * \emptyset\left\|y-Y^{j}\right\|\right)
$$

\subsection{Genetic Algorithm (GA)}

GA consists of a population propped for selection via evolution principles so that each potential solution is an individual for which optimal is found using four operators as below (Coello et al, 2004 and Reynolds, 1994). Its fitness function determines how close an agent is to optimal solution so that agents that are close to its fitness value are said to be fit. The operators include (Ojugo et al, 2013a,b):

a. Initialize - Data is encoded into forms suitable for selection. Each encodings type has its merit. Binary encoding is computationally more expensive. Decimal encoding has greater diversity in chromosome and greater variance of pools generated; float-point encoding or its combination is more efficient than binary. Thus, it encode as fixed length vectors for one or more pools of different types. The fitness function evaluates how close a solution is to its optimal - after which they are chosen for reproduction. If solution is found, function is good; else, is bad and not selected for crossover. The fitness function has knowledge of task. If more solutions are found, the higher its fitness value.

b. Selection - best fit individuals close to optimal are chosen to mate. The larger the number of selected, the better the chances of yielding fitter individuals. This continues until one is chosen, from the last two/three remaining solutions, to become selected parents to new offspring. Selection ensures the fittest individuals are 
chosen for mating but also allows for less fit individuals from the pool and the fittest to be selected. A selection that only mates the fittest is elitist and often leads to converging at local optima.

c. Crossover ensures best fit individual genes are exchanged to yield a new, fitter pool. There are two crossover types (depends on encoding type used): (a) simple crossover for binary encoded pool. It allows single- or multi-point cross with genes from a parent, and (b) arithmetic crossover allows new pool to be created by adding an individual's percentage to another.

d. Mutation alters genes by changing its sequence, to ensure a new pool converges to global minima (instead of local optima). Algorithm stops if optimal is found, or after a number of runs creates new pool (this computationally expensive), or when no better solution is found. Genes may change based on probability of mutation rate. Mutation improves the much needed diversity in reproduction and its algorithm.

\subsection{Data Sampling and Collection}

A critical feat in ANN design is its dataset size and frequency. This affects its final result. For short-term forecast, high frequency data is preferred (i.e. daily or intraday) though not always available and very costly. Thus, we use weekly/monthly data because it is less noisy. A second important feat is data coverage (Kulkarni and Haidar, 2009). The more data point is used in ANN, the better its generalization. Some modelers discard older dataset when dealing with financial data as economic conditions change over time. Smith (1993), McNeils (2005) and Kulkarni and Haidar (2009) believe that training ANN with old irrelevant data alongside current conditions can result in poor model generalization. However, we believe that broadening our data coverage helps ANN avoid pitfalls of over-parameterization, overtraining and over-fitting. OPEC data is available at:

http://investexcel.net/opec-basket-histor-excel.htm.

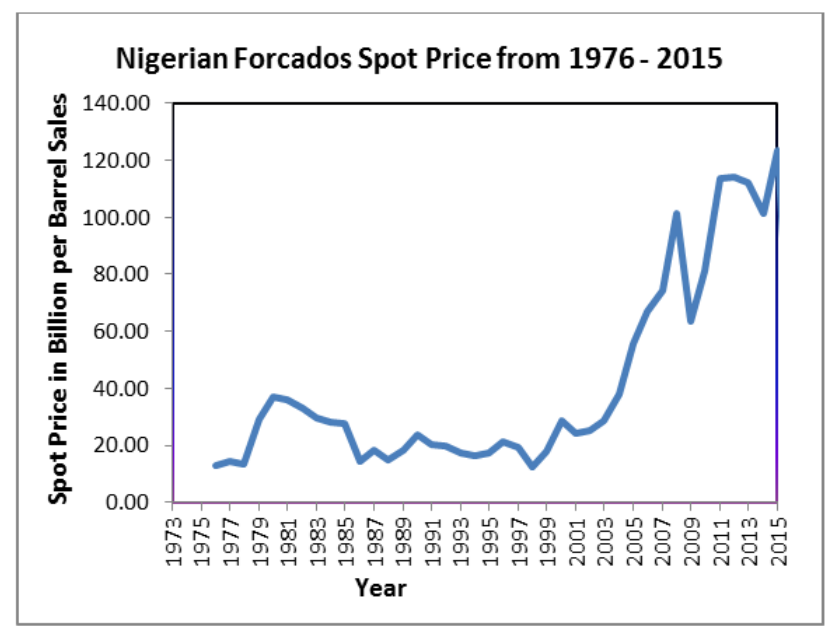

Figure 1. Nigerian Forcados Spot Price with Date.

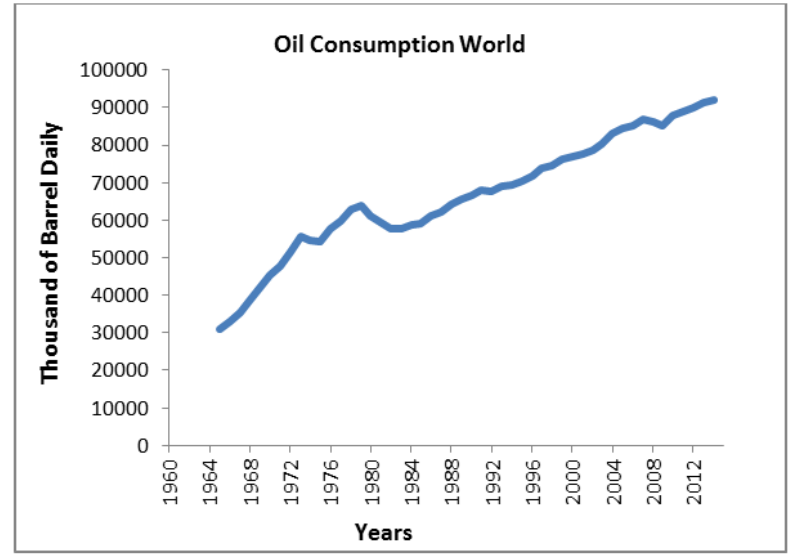

Figure 2. Worldwide Consumption of Oil.

The study is limited to secondary data obtained from The Energy Information Administration on monthly all countries spot price and consumption of crude oil from March 1976 December 2015. The GANN model is used in the computation of the price direction and implied volatility used to determine the fluctuations in price direction; while some other trading strategies used in hedging commodities like crude oil are looked at.

\subsection{Rationale for the Model}

1. The Gabillon Model provides a good start point (initial) solution for the GANN model - as all data are treated as new in feedforward network and thus, previous data do not help identify data feats of interest (in this case, accurate price and volatility forecast) even if such observed datasets exhibits temporal dependence.

2. The structural dependencies imposed by dataset and by adopted hybrid methods, on the model, is resolved via its ability to store earlier generated data from previous layer(s), unlike feedforward networks that must be expanded and extended to represent such complex, dynamic patterns and scenario as this (Kuan, 1994; Ojugo et al, 2013).

3. As network becomes larger and more data are fed in, feedforward net makes it practical difficult to implement. Jordan net overcomes this difficulty via its internal feedbacks that inputs back its output into the hidden unit with a time delay so that output at $t+1$ becomes input at later $\mathrm{t}$ - making it better suited for such task.

4. Jordan net is more powerful and computationally more plausible. Its backpropagation-in-time algorithm uses output at $t$, as input alongside new input to compute output at $t+1$ in response to market data changes (Mandic and Chambers, 2001) via Tansig activation function $\mathrm{y}^{\mathrm{k}}$. It sums all input, receives target value of input patterns, compute error data, weight correction updates in layers $\left(c_{j}^{k}\right)$ and bias weights correction updates $\left(c_{o}{ }^{k}\right)$. Its error is sent back from output layer into input nodes via error backpropagation (to find weights that estimates target output with selected accuracy) to correct its weights. Weights are modified by minimizing 
error between target and computed outputs, at the end of each forward pass. If the error is higher than selected value, process continues with a reverse pass; else, training stops. Weights are updated via mean square error until a minimal error is achieved (Ursem et al, 2002).

\section{Experimental Model}

Our model hinges on 3-basic methods: (a) Gabillon model, (b) Jordan neural network, and (c) Genetic algorithm as in figure 2. Data pre-processing is quite a sensitive task as it often destroys the inbuilt structure in an original dataset (Azoff, 1994; Vanstone, 2005). Thus, as opposed to the use of a weak stationary process, we implement thus:

1. A major feat of ANN is in its learning ability with advanced algorithms such as backpropagation with momentum to resolve issues in using a weak stationary process as justified by Kulkarni and Haidar (2009).

2. The idea and behaviour of the oil market that is rippled with shock and fluctuations (due to volatility) is the focal point and reason for predicting price direction. This, allows participants to classify contango and backwardation. As such, proper modeling and parameterization of the oil market is critical such that the dataset must reflect such behaviours too.

3. Replacing a non-stationary, dynamic data with weak-stationary one will lead to false-positives and true-negatives result, which will also mislead market participants and policymakers.

4. Influence of shock cannot be overemphasized; And though, it lingers over various steps, forecast accuracy must not be trade-off for easy implementation and likely agreement of result.

5. Using non-stationary data makes easier ANN to estimate general characteristics and feats in dataset; rather, than actual relationship (Refenes, 1995). Thus, data looses originality, quality and inbuilt structures via such transformation advocated in Kulkarni and Haidar (2009) since forecasts retrieved overtime as result has the same nature and structure required as output for forecasting price direction and volatility.

The market as a model that allows data (historical, current and update samples) as input so that market participants react based on their dispositions, speculations, analysis etc to result in an output closing price for an asset, which is an aggregation of their activities. Imitating the market, our model tries to map the adopted available data to desirable target so that its forecast is with a certain degree of accuracy and an acceptable degree of error (Refenes, 1995).

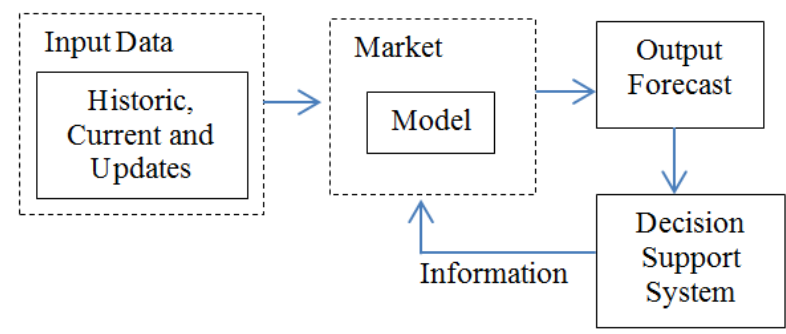

Figure 3. Modified Model selection to mimic the Market.

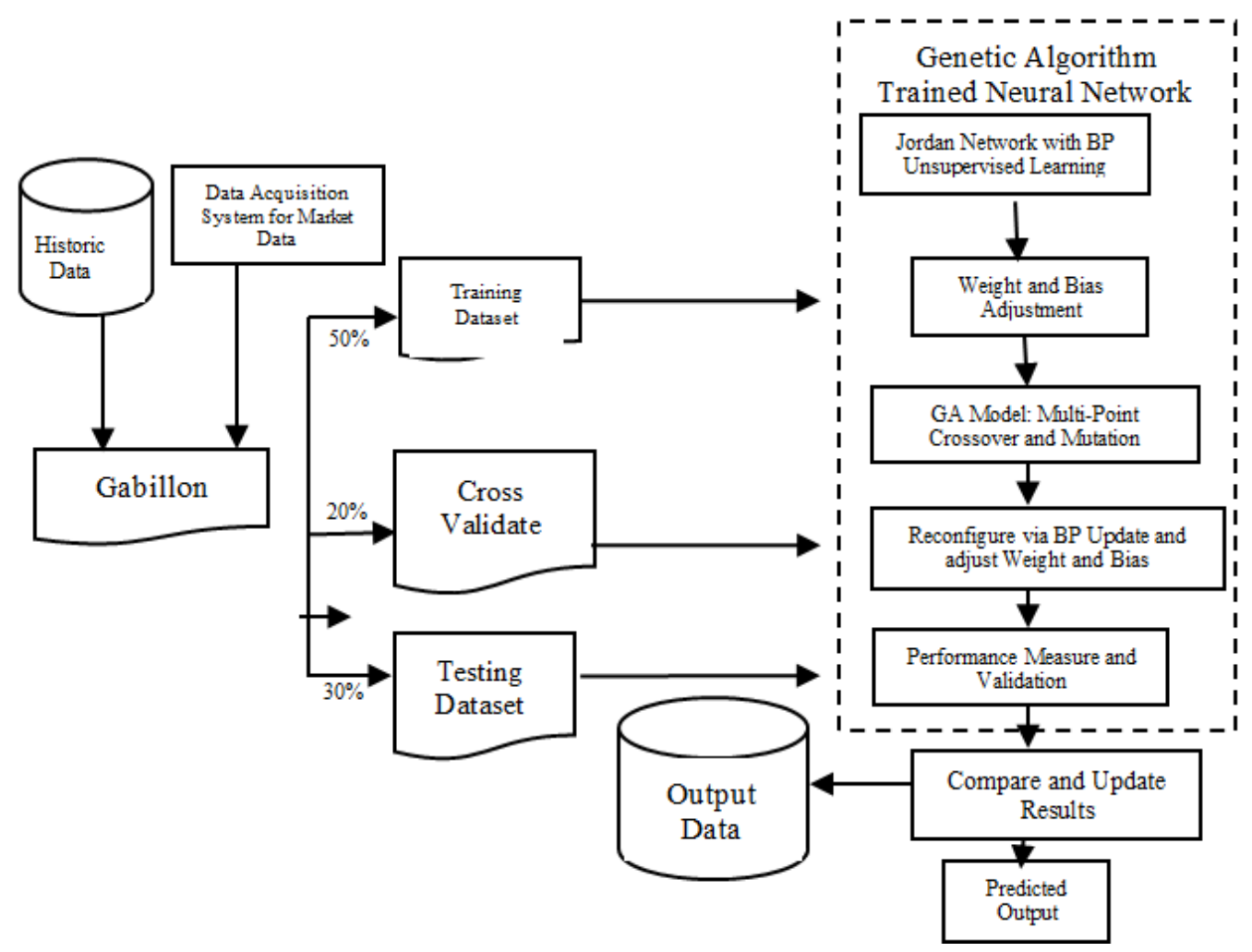

Figure 4. Dataflow Diagram of the Hybrid Genetic Algorithm Trained Neural Network. 
We use ANN as nonparametric and nonlinear mapping-model (Azoff, 1994) with no prior assumption of task - so that the sample updates used as data coverage is expanded will help model recall price direction (contango/backwardation) for better forecast. And the dataset is harnessed to speak for itself (Azoff, 1994). Unlike Kulkarni and Haidar (2009), we adopt a recurrent ANN (as better suited to estimate nonlinear, continuous function) to forecast oil prices direction, test if futures prices with newer data predicts price direction in near futures and if data in futures price integrated with spot-price yields better forecast with much attention paid to finding an optimal solution in the GANN model, which will seek to harmonize a benchmark from current and past data embedded in futures price as in figure 4 .

\subsection{Modeling}

The proposed experimental model is employed as:

a. First, we use the Gabillion model as a preprocessor classifier to help forecast futures prices and volatilities of contracts in the oil market. This will in turn propagates the values of selected data as further input to the model as enhanced, defined variable classes that are partitioned into data-points.

b. Dataset is grouped into: training, retraining and testing, and used to initialize the GANN model. The Jordan net is an unsupervised, self-learning model whose optimization is achieved via GA's recombination and mutation. For our Jordan net, we use a multilayered perceptron (feedforward) net with short-memory (i.e. time-lag network) with local recurrent connections, which requires a smaller network to learn temporal task. This is because - irrespective of how large the network and data grows, it is more plausible and computationally more powerful than other adaptive models as it uses backpropagation-in-time and momentum learning to train the network so that output at $t$ is used along with new data to compute output at $t+1$ in response to the phenomenon's dynamism and chaotic nature.

GA recombines and mutates the dataset so that model autonomously forecasts futures price. Model is initialized for data selection via the preprocessor Gabillon model. Knowledge of the task has direct impact on how model processes the data, determines how close a solution is, and how the algorithm is employed. Model stops as best individual has a fitness of 0 (Campolo et al, 1999; Dawson and Wilby 2001b). Our ANN uses BP-in-time and momentum learning, which uses error derivative propagated back to neurons to adjust its weight updates as thus (Ojugo et al, 2015a,b,c):

1. Set all weights to small random values.

2. Input to each node: $x_{i}$ input from previous node, $w_{i}$ is weight - so that sigmoid function computes thus:

$$
\begin{gathered}
\text { Input } \alpha=\in X_{i} W_{i} \\
\text { Output } Y=f(x)=\frac{1}{(1+e-x)}
\end{gathered}
$$

3. Its errors, desired and actual output is sent back to nodes with updated weights via Eq. $10\left(\mathrm{w}_{\mathrm{ij}}\right.$ is weight from node $i$ to $j$ at $t, \mathrm{y}$ is learning rate, oj is output of $\mathrm{j} ; \mu \mathrm{j}$ is error for node j). So:

$$
\begin{aligned}
& W_{i j}(t+1)=W_{i j}+\eta \mu j o j \\
& \text { Output node: } \mu j=k o j(1-o j)(t j-o j)
\end{aligned}
$$

Hidden nodes with $\mu_{\mathrm{k}}$ as next nodal error term:

$$
\mu j=k o j(1-o j) \in \mu k \cdot w j k
$$

c. Thirdly, we initialize GANN with dataset from Gabillon preprocessor model so that agents are selected from the pool via a tournament method. The tournament method is used because it is easier, more efficient to code, best suit for parallel architectures and its selection is easily adjusted as it allots random numbers to agents. Their fitness function is computed to determine mating agents and their corresponding solutions so that they are made available as a new pool from single parent. We use a multipoint crossover to introduce chaos and volatility as in the market. Mutation helps the network to learn all non-linear, dynamic feats in dataset. With agents in original pool from single parent, network uses Gaussian distribution to randomly generate data corresponding to crossover points. And as new parents contribute to pool, it yields new agents whose genetic makeup is a combination of both parents. Mutation is applied to yield agents that also undergo further mutation that re-allocates to them new random values. The number of mutation applied depends on how far GA progresses on the model (i.e. how fit the fittest agent is in the pool). This equals the fitness of the fittest individual divided by 2. New agents are selected to replace old ones of low fitness - to create a new pool. The process continues until individual with a fitness value of 0 is found indicating solution has been reached (Branke, 2001).

\subsection{Model Performance}

We evaluate model's performance via its computed coefficient of efficiency (COE), mean square error (MSE), mean absolute error (MAE) and mean relative error (MRE), and $\operatorname{COE}(\mathrm{R})$ to show the model's efficiency. A model with minimum error is considered, best choice.

$$
\begin{aligned}
M S E & =1 / n \sum_{i=1}^{m}\{(\text { Ypi }- \text { Yio }) 2\}^{1 / 2} \\
M A E & =1 / n \sum_{i=1}^{m} \mid \text { Ypi }- \text { Yio } \mid \\
M R E & =1 / n \sum_{i=1}^{m} \frac{\mid \text { Ypi-Yio } \mid}{\text { Yio }}
\end{aligned}
$$

Model validation should not be performed by single researcher or research group; But rather, a scientific dialogue as the improper model application along with its ambiguous results often presented by modelers that impedes such dialogue. The aim of this hybrid thus, is to a great extent 
minimize and reduce the confusion in financial data time-series. We use the MLP model as a benchmark to measure our model performance.

Table 1. Comparative Model Performance.

\begin{tabular}{|c|c|c|c|c|}
\hline \multirow{2}{*}{ Model } & \multicolumn{4}{|l|}{ Training } \\
\hline & COE (R) & MSE & MAE & MRE \\
\hline GGANN & 0.982 & 0.282 & 0.328 & 0.213 \\
\hline MLP & 0.515 & 0.4309 & 0.665 & 0.8708 \\
\hline \multicolumn{5}{|c|}{ Cross-Validation } \\
\hline Jordan & 0.892 & 0.329 & 0.231 & 0.1901 \\
\hline MLP & 0.512 & 0.686 & 0.712 & 1.109 \\
\hline \multicolumn{5}{|l|}{ Testing } \\
\hline Jordan & 0.966 & 0.296 & 0.219 & 0.1710 \\
\hline MLP & 0.641 & 0.654 & 0.518 & 1.385 \\
\hline
\end{tabular}

The model performance table as above implies that forecast of futures prices with newer data yields efficient price direction in near futures. The expansion of our data coverage reveal also that spot-price integrated with futures price yields better forecast with much attention paid to finding an optimal solution in the GANN model.

\subsection{Experimental Findings and Analysis}

Though recurrent net takes time to converge; Our hybridization help speed ANN process so that model converge after 3 minutes and 29seconds with 500 iterations on stand-alone machine. While, network keeps various numbers of previous steps in memory (context) layer, its result varies for each retraining time-step and is in tandem with the chaotic and dynamic conditions of the dataset and volatility. Also, though the Jordan $\mathrm{NN}$ require more hidden neurons to converge due to context layer, our use of MLP with momentum - works in place of it. The choice of activation function, weight/bias, and learning rate are determined by the experiments. The model leads to fast convergence, and higher hit rate.

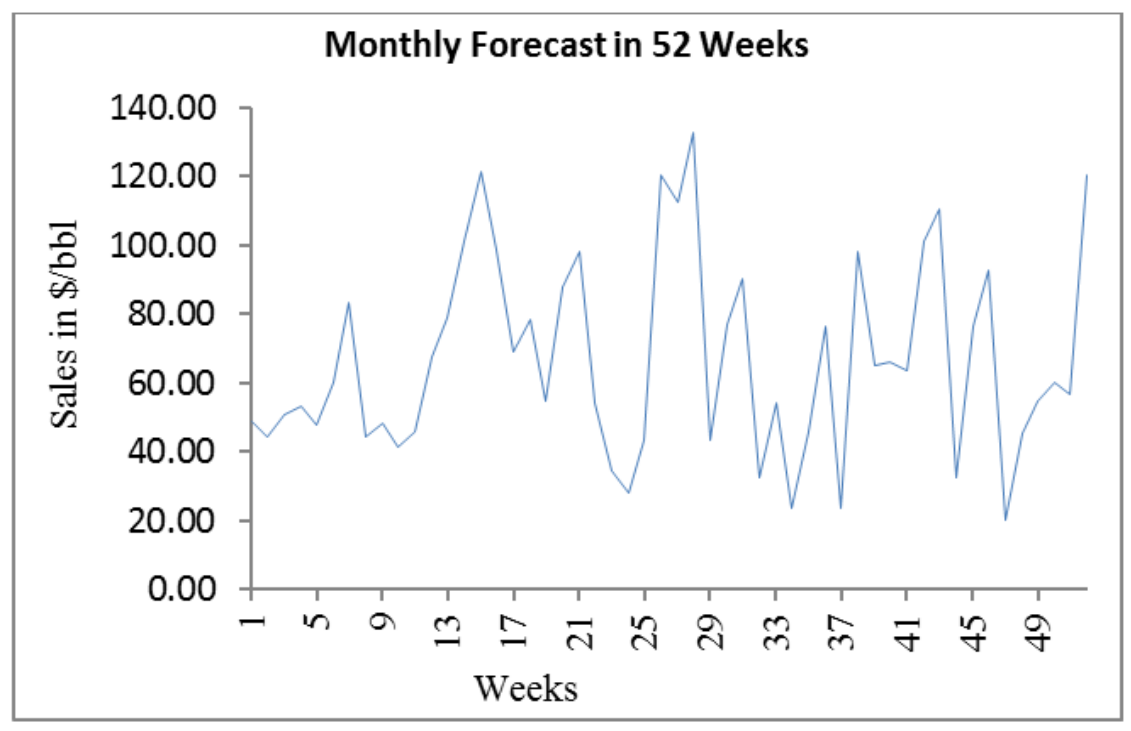

Figure 5. Futures price direction and volatility.

Figure 5 show futures price direction monthly forecast for 2016 and 2017. The spot price for each month is monthly average oil price (dollars per barrel) and its volatility is estimated from prices in previous year. For 2016, volatility vary between 1.9012 and 0.312; And for 2017, volatility varies between 0.16 and 0.3542 for 12-months (52 weeks) futures maturity. Thus, the oil price still go up due to demand; Rather, than plummet in the near future. However, energy is about dominance and a lot of international politics are displayed when energy is concerned due to vested interests. These result in various shocks ranging from convenience yield etc.

Also, studies of oil price direction emphasize the role of interest rates and convenience yield (adjusted spot-futures spread) to confirm that spot price normally exceed discounted futures. Though most studies do not explain why such 'backwardation' is normal, it is a result of hedging and speculations. We also noted it is far better to hold a physical asset than hold futures contracts as proposed by hedging. Also, convenience yield behaves nonlinearly; And, price response to convenience yield is also nonlinear. Thus, futures price are informative about future spot prices only when spot prices substantially exceed futures.

Producer hedging is observed as a way forward such that if spot-price of oil is $\$ \mathrm{x} / \mathrm{barrel}$ at $t$ and producer expect price to fluctuate between $t$ and $\mathrm{T}$ (maturity time for hedge). If producer is more concerned about risk of prices falling below $\$ y /$ barrel and prepared to accept maximum price of $\$ \mathrm{z} /$ barrel. Hedging allows participants to buy at \$y/barrel put and sell at \$z/barrel call. This limits backwardation (downside) and contango (upside) price risks to the range between $\$ y /$ barrel and $\$ z /$ barrel. If oil prices falls below $\$ y /$ barrel at $t, \$ z$ call option is worthless and the \$y put option is exercised to grant the producer the right to sell its output at $\$ y /$ barrel (no matter how low prices go). If prices rise over \$z/barrel, the \$y put option becomes worthless as $\$ z$ call option is exercised and producer will sell at $\$ \mathrm{z} /$ barrel (no matter how high prices go). But, if prices are between \$y/barrel and \$z/barrel, neither of the options is exercised so that the producer sells at the 
prevailing market price. This is known as a collar. The strike price of the option can be set at any level, but the put and call options must be equally far out-of-the money if the cost of the put and call is to be the same. If the costs of the options are the same, the strategy is known as a zero collar (Cherry, 2007; Dontwi, Dedu and Davis, 2010).

\section{Conclusion}

The following recommendations were made:

1. Nigeria is a mono-product, reactive nation that solely depends on Oil. Her politics/policies are enmeshed in price fluctuations. Thus, her policymakers must devote great effort towards regular updates in price change for effective politics/policy implementation as she seeks new funding alternative and become forward thinking. Thus, she must open her investment as well as advance innovative research to improve her local contents and human capital. The futures prices will continue to fluctuate plus, no sane economy effectively plans with such volatility as non-OPEC members will continue to sell at cheaper prices just to dispose their commodity.

2. Energy is about dominance, and nations seek to less depend on commodities that enslave them to other nations as possible. Various system to help us live better with cleaner environ depends on Oil. So be it oil or its alternative, energy will continue to experience structure price fluctuations and volatility because nations will seek to be less dependent on others via enforced use of their local products.

3. Our analysts should always strategize to keep up with new innovations and shifts in paradigm from one source of energy to another. So that with a plummet or outright non-dependence, we have to be prepared for such outcomes.

4. Cutting edge innovation will always advance as vehicles for great change. Nigeria cannot be left out in this change Our dependence on oil has led us to recession. Government must harness the plethora of tools, analysts and academia at their disposition for better productivity. Singapore as a nation has no single natural resources; Yet, today - their standard of living as proven by financial times and fox business - exceeds that of the United Kingdom. Japan closed her borders to all form of imports and today, they are a super nation. China, still invests heavily on human capital. Human capital development cannot be over-emphasized as it is the key to innovation, production of any kind and real progress and advancement.

Oil is vital for economic growth in both industrialized and developing nations. And the oil market is engulfed and endangered by speculations in the finance market, politics, extreme weather phenomena, among others - which accounts for its increased price fluctuations and volatility. The price fluctuation effect extends its influence over a large number of goods and services with direct impact on economies. Thus, to reduce its negative impact, it is imperative to predict price direction(s) regularly. But, some fundamental parameters (such as oil supply, demand inventory, GDP, seasonal data, jumps and spikes etc) are not readily available on daily scale. These dynamic and chaotic events all adds to the complexity involved in the prediction of oil price and volatility (Kulkarni and Haidar, 2009).

Focus on models to predict futures price direction and volatility must continue, since futures price reacts faster to new data than spot price. Such forecast allow investors to harness the many benefits of the market such as low transaction cost, high liquidity, premium etc. Such infusion of new-market-related data help investors dispose themselves to either buy or sell in the market (Kulkarni and Haidar, 2009), and empirical evidences provided beckon on investors to harness the many merits of the model without trading a physical asset; But, rather using only contracts or bonds (Ojugo, 2016).

To judge the price implication of fitting structural models, or apply it as symbiotic informed decision - on other assets all of which have different market structures and fundamentals, is time-consuming, non-cost effective and may not add no more value than just being an extrapolation from the current or spot price.

\section{References}

[1] Abosedra, S and Baghestani, H., (2004). On predictive accuracy of futures prices, Energy Policy, pp. 1389-1393.

[2] Abraham, A., (2005). Handbook of Measuring System Design, John Wiley and Sons Ltd, ISBN: 0-470-02143-8, pp. 901-918.

[3] Azoff, M., (1994). Neural network time series forecasting of financial markets, John Wiley \& Sons, Chichester.

[4] Behmiri, N. B and Manso, J. R. P., (2013). Crude oil forecasting techniques: a comprehensive review of literature, Alternative Investment Analyst Review in Social Science Review Network, doi: 10.2139/ssrn.2275428, 30-48

[5] Beven, K. J and Binley, N. J., (1992). Knowledge driven models and computations, Wiley and Sons, Chichester, ISBN: 0-465-08245-3.

[6] Bishop, C. M., (1995). Neural networks for pattern recognition, Oxford University Press, ISBN: 0-342-45230, pp.253-294.

[7] Bopp, E and Sitzer, S., (1987). Are petroleum futures prices good predictors of cash value?, Journal of Futures Market, 705-719, 1987.

[8] Branke, J., (2001). Evolutionary Optimization in Dynamic Environments, Kluwer.

[9] Brooks, C., (2002). Introductory econometrics for finance, Cambridge University press, Cambridge.

[10] Brooks, C., Rew, G., Alistair, S and Ritson, S., (2001). A trading strategy based on lead-lag relationship between the spot index and futures contracts for FTSE 100, International Journal of Forecasting, 17: 31-44.

[11] Campolo, M., Andreussi, P and Soldati, A., (1999). Flood forecasting with a neural network, Water Resources, 35(4): 1191. 
[12] Caudill M., (1987). Neural Networks Primer, AI Expert December, pp. 46-52.

[13] Chan, K., (1992). A further analysis of the lead-lag relationship between the cash market and stock index futures market, The Review of Financial Studies, 7(6): 123-152.

[14] Chakroborty, A. L., (2010). Introductory artificial intelligence, Lecture note on Computer Science, Massachuset Institute of Technology, [online]: www.mit.courseware

[15] Cherry, H. (2007). Financial Economics, 1st Ed. Actuarial Study Materials, NY: 3217 Wynsum Ave., Merrick.

[16] Coello, C., Pulido, G and Lechuga, M., (2004). Handling multiple objectives with particle swarm optimization, In Proceedings of Evolutionary Computing, Vol. 8, pp 256-279.

[17] Coppola, A., (2007). Forecasting oil price movements: exploiting the information in futures market, 34, http://papers.ssrn.com/paper.taf?paper_id $=967408$

[18] Dawson, C and Wilby, R., (2001a). Comparison of neural networks in river flow forecasting, J. of Hydrology and Earth Science, SRef-ID: 1607-7938/hess/2001-3-529.

[19] Dawson, C and Wilby, R., (2001b). Hydrology modeling using neural network, Journal of Programming in Physics and Geography, 25: 80-108.

[20] Dontwi, I. K., Dedu, V. K and Davis, R., (2010). Application of options in hedging of crude oil price risk, American Journal of Social and Management Sciences, ISSN: 2156-1540, doi: 10.5251/ajsms.2010.1.1.67.74.

[21] Fausett L., (1994). Fundamentals of Neural Networks, New Jersey: Prentice Hall, pp.240.

[22] French, M. W., (2005). Why and When do Spot Prices of Crude Oil Revert to Futures Price Levels?, Finance and Economics Discussion Series Divisions of Research and Statistics and Monetary Affairs, Federal Reserve Board, Washington, D. C.

[23] Gabillon, J., (1991). Terms of oil future prices, Oxford institute of Energy Studies, ISBN: 0-948061-59-6

[24] Heppner, H and Grenander, U., (1990). Stochastic non-linear model for coordinated bird flocks, In Krasner, S (Ed.), The ubiquity of chaos (pp.233-238). Washington: AAAS.

[25] Karunanithi, N., Grenney, W., Whitley, D and Bovee, K., (1994). Neural network for river prediction", Journal of Computing in Civil Engineering, 8(2), pp.201-220.

[26] Kulkarni, S and Haidar, I., (2009). Forecasting model for crude oil price using artificial neural networks and commodity futures prices, International Journal of Computer Science and Information Security, 2(1): 38-49.

[27] Labonte, M., (2004). The effect of oil shocks on the economy: A review of the empirical evidence, RL31608.

[28] Laurenti, M and Fernandes, J. M. M., (2012). Pricing crude oil calendar spread option, Master's Degree Thesis, Department of Finance, Copenhagen Business School.

[29] Mandic, D and Chambers, J., (2001). Recurrent Neural Networks for Prediction: Learning Algorithms, Architectures and Stability, Wiley and Sons: New York, pp56-90.

[30] McNeils, D., (2005). Neural networks in finance gaining predictive edge in the market, Elsevier Academic Press, Massachusetts.

[31] Minns, A., (1998). Artificial neural networks as sub-symbolic process descriptors, published $\mathrm{PhD}$ Thesis, Balkema, Rotterdam, Netherlands.

[32] Moshiri, S and Foroutan, F., (2005). Forecasting nonlinear oil futures prices, The Energy Journal, 27: 81-95.

[33] Ojugo, A. A, (2012). A hybrid neural networks trained gravitational search algorithm for rainfall runoff modeling, $\mathrm{PhD}$ Thesis, CS Department: Ebonyi State University Abakiliki, Nigeria.

[34] Ojugo, A. A, (2016). Spread options pricing and hedging for Oil market: a case of OPEC, Lecture Series of the Federal University of Petroleum Resources Effurun, Nigeria.

[35] Ojugo, A. A., and Yoro, R., (2013a). Computational intelligence in stochastic solution for Toroidal Queen, Progress in Intelligence Computing and Applications, 2(1), pp 46-56.

[36] Ojugo, A. A and Ofualagba, G. O., (2016). Repositioning FUPRE Petroleum Varsity to tackle challenges in the oil and gas sector of Nigeria, Guardian Newspaper.

[37] Ojugo, A. A., Emudianughe, J., Yoro, R. E., Okonta, E and Eboka, A. O., (2013b). Hybrid neural network gravitational search algorithm for rainfall runoff modeling, Progress in Intelligence Computing and Application, 2(1), doi: 10.4156/pica.vol2.issue1.2, 22-33.

[38] Ojugo. A. A., A. O. Eboka., R. E. Yoro., M. O. Yerokun., F. N. Efozia., (2015). Hybrid model for early diabetes diagnosis, Mathematics and Computers in Science and Industry (A Mathematics and Computers in Science and Engineering Series), 50: 176-182, ISBN: 978-1-61804-327-6, ISSN: $2227-4588$.

[39] Ojugo. A. A., A. O. Eboka., R. E. Yoro., M. O. Yerokun., F. N. Efozia., (2015). Framework design for statistical fraud detection, Mathematics and Computers in Science and Industry (Mathematics and Computers in Science and Engineering Series), 50: 176-182, ISBN: 978-1-61804-327-6, ISSN: 2227-4588.

[40] A. A. Ojugo., I. J. B. Iyawa., F. O. Aghware., M. O. Yerokun., E. Ugboh., (2015). Comparative study of timetable constraint satisfaction problem, Latest trends in Circuits, Systems, Signal Processing and Automatic Control, 34: 176-182, ISBN: 978-960-474-374-2, ISSN: 1790-5117.

[41] Perez, M and Marwala, T., (2011). Stochastic optimization approaches for solving Sudoku, IEEE Transaction on Evol. Comp., pp.256-279.

[42] Rajurkar, M. P., Kothyari, U and Chaube, U., (2004). Modeling of daily rainfall-runoff relationship with artificial neural network, Journal of Hydrology, Vol. 28, 96-113.

[43] Refenes, A. A., (1995). Neural networks in the capital markets, John Wiley and Sons, New York.

[44] Reggiani, P. and Rientjes, T., (2005). Flux parameterization in the representative elementary watershed approach: Application to basin Water Resources, 41(4), pp 18-27.

[45] Reynolds, R., (1994). Introduction to cultural algorithms, Transaction on Evolutionary Programming (IEEE), pp.131-139. 
[46] Rouah, F. D and Vainberg, G., (2007). Option pricing and volatility using Excel VBA, John Wiley and Sons, NY, ISBN: 978-0-471-79464-6.

[47] Sharma, N., (1998). Forecasting oil price volatility, Masters of Art Thesis in Economics, Virginia Polytechnic Institute and State University.

[48] Silvapulle, P and Mossa, A., (1999). The relation between spot and future prices: Evidence from the crude oil market, The Journal of Futures Markets, 19(2), 175-193.

[49] Smith, M., (1993). Neural networks for statistical modeling, Van Nostrand Reinholo, New York.
[50] The International Energy Agency (2009) Analysis of the impact of high oil prices on the global economy, Annual Report.

[51] Ursem, R., Krink, T., Jensen, M. and Michalewicz, Z., (2002). Analysis and modeling of controls in dynamic systems. IEEE Transaction on Memetic Systems and Evolutionary Computing, 6(4): 378-389.

[52] Vanstone, B., (2005). Trading in Australian stock market using artificial neural networks, McGraw Hill publication.

[53] Verleger, P. K. (1993). Adjusting to Volatile Energy Prices, Institute for International Economics, Washington DC. 